\title{
La tesis del caso especial *
}

\author{
ROBERT ALEXY \\ Universidad de Kiel
}

\begin{abstract}
A partir de la comprensión de la tesis del caso especial como un intento por dar cuenta de la doble vertiente de la argumentación jurídica (su carácter autoritativo, instítucional o real, por un lado, y su dimensión libre, discursiva o ideal, por otro) Alexy se propone defender esta tesis frente a las críticas tomadas en consideración o realizadas por Jürgen Habermas en su libro Facticidady validez. En primer lugar, Alexy defiende su tesis frente a la critica que sostiene que las limitaciones que existen en los procedimientos judiciales excluirian su comprensión en términos de teoría del discurso. En
\end{abstract}

segundo lugar, el autor hace hincapié en que la tesis del caso especial que él defiende implica que la argumentación jurídica ha de entenderse como un caso especial del discurso práctico general y no meramente del discurso moral tal y como es definido por Habermas. $Y$ por último, defiende la tesis del caso especial contra la objeción de que los argumentos prácticos generales cambjarian su carácter al pasar a formar parte del Derecho; para ello propugna concebir de manera procedimental la integración de los argumentos prácticos generales en el contexto jurídico.

\section{EL CARÁCTER AUTORITATIVO Y DISCURSIVO DEL RAZONAMIENTO JURÍDICO}

La teoría discursiva del Derecho comprende un conjunto de temas que van desde el problema del conocimiento práctico hasta la teoría de la democracia, pasando por el sistema de derechos. Entre estas materias, la teoría de la argumentación jurídica se encuentra más cerca de la práctica jurídica. Esto le permite convertirse cn una especie de piedra de toque para la corrección de la sobrearqueada idea de la racionalidad discursiva en el Derecho. La teoría de la argumentación jurídica puede desempeñar este papel porque se encuentra intrínsecamente conectada con todos los elementos del sistema jurídico. Dos ejemplos pueden servirnos de ilustración. El primero se refiere a la relación entre el proceso democrático y la argumentación jurídica. El proceso democrático, que tiene por resultado las decisiones parlamentarias, suministra los puntos de partida más importantes para la argumentación jurídica en un Estado constitucional democrático: las leyes. El segundo ejemplo serian los derechos fundamentales. No basta con que un proceso constitucional concluya con la adopción de un catálogo de derechos fundamentales; éstos tienen que ser interpretados e implementados. Esto puede ser llevado a cabo, en parte, por la

\footnotetext{
* La traducción del original inédito inglés ha sido realizada por Isabel Lifante.
} 
legislatura; pero si el propio legislador está sometido a los derechos fundamentales, deberá existir un tipo de argumentos sobre si un acto u omisión legislativa viola o no los derechos fundamentales. Los argumentos que interpretan los derechos fundamentales de una determinada constitución son argumentos jurídicos, de manera que existe una relación necesaria entre los derechos fundamentales, que vinculan a todos los poderes del Estado, y la argumentación jurídica.

Estos dos ejemplos muestran que la argumentación juridica tiene una doble vertiente. Por un lado, participa intensamente en el carácter autoritativo, institucional o real del Derecho. Esto puede observarse en el papel que las razones autoritativas desempeñan en los argumentos jurídicos y en el marco institucional del razonamiento jurídico, papel que conduce, en última instancia, no sólo a sugerencias y propuestas, sino también a decisiones definitivas de los tribunales, impuestas, si fuera necesario, por el poder. Por otro lado, el razonamiento jurídico permanece estrechamente conectado con lo que puede llamarse la vertiente libre, discursiva o ideal del Derecho. La necesidad de razonamiento jurídico, como algo más que la mera subsunción y, en cuanto tal, más que la mera ejecución de lo autoritativo, surge de la frecuentemente mencionada textura abierta del material autoritativo (Hart, 1994, 126 y ss.). El razonamiento en defecto de material autoritativo puede, por definición, no estar determinado únicamente por lo que es autoritativo. Este razonamiento puede resultar libre hasta un cierto grado. Por lo que respecta a los precedentes, la libertad es incluso mayor. Al margen de su mayor o menor carácter autoritativo, todo el mundo es libre para criticar las decisiones judiciales con argumentos jurídicos. Tal crítica puede llevar a la revisión de una sentencia por un tribunal superior o a su revocación por un tribunal que, posteriormente, decida un caso similar. Una buena teoría de la argumentación jurídica debe dar cuenta tanto de la vertiente autoritativa, institucional o real del razonamiento jurídico como de su dimensión libre, discursiva o ideal. La tesis del caso especial (Sonderfallthese) que es objeto de mis consideraciones es, precisamente, un intento de conseguirlo.

\section{LA TESIS DEL CASO ESPECIAL}

La tesis del caso especial establece que el discurso jurídico es un caso especial del discurso práctico general (Alexy, 1989a, 205 y ss.). Se basa en tres razones. La primera radica en que la discusión jurídica, al igual que la argumentación práctica general, se refiere, en definitiva, a lo que es obligatorio, prohibido o permitido, es decir, a cuestiones prácticas. La segunda razón consiste en que $\mathrm{cn}$ el discurso jurídico surge, al igual que en el discurso práctico general, una pretensión de corrección. Ambos tipos de argumentaciones son, por tanto, discursos. La tercera razón establece que la argumentación jurídica es un supuesto de caso especial, porque la pretensión de corrección del discurso jurídico 
es distinta a la del discurso práctico general. No se refiere a lo que es absolutamente correcto, sino a lo que es correcto en el esquema y con las bases de un orden jurídico válidamente imperante. Lo que es correcto en un sistema jurídico depende esencialmente de lo que es fijado autoritativa o institucionalmente y de lo que encaja con ello. No debe contradecir lo autoritativo y debe ser coherente con el conjunto. Para expresarlo brevemente podría decirse que la argumentación jurídica ha de estar vinculada a las leyes y a los precedentes y tiene que observar el sistema de Derecho elaborado por la dogmática jurídica.

\section{OBJECIONES}

Se han dirigido muchas críticas contra la tesis del caso especial. No intentaré ocuparme ahora de todas ellas. Algunas ya han sido discutidas en otras ocasiones (cfr. Alexy, 1993, 157 y ss.; 1989a, 291 y ss.). Aquí me concentraré en las objeciones tomadas en consideración o rcalizadas por Jürgen Habermas en su libro Faktizität und Geltung, publicado en castellano como Facticidad y validez.

\section{Los procedimientos judiciales}

La tesis del caso especial puede ser relativa tanto a los procedimientos judiciales como a la argumentación jurídica en cuanto tal, esto es, la argumentación jurídica tal como tiene lugar, por ejemplo, en los libros, artículos o discusiones académicas. La primera se encuentra institucionalizada, mientras que la segunda no. Esta última tiene el carácter abierto e infinito típico de las discusiones científicas. Esto la hace bastante diferente de los procedimicntos judiciales definidos por las reglas procedimentales que gobiernan tanto la acción forense de las partes como la del propio tribunal. Algunos autores han argumentado que estas limitaciones excluyen la comprensión de los procedimientos judiciales en términos de teoría del discurso (Neumann, 1986, 84 y s; Kaufmann 1989, 20 y ss.), pudiendo aludir a varios rasgos de tales procedimientos que a primera vista parecen incompatibles con cualquier conexión entre ellos y los discursos. Valgan como ejemplos la distribución asimétrica de roles en los procesos penales, las limitaciones de tiempo o las motivaciones reales de los participantes, quienes a menudo (si no generalmente) pretenden conseguir un juicio favorable y no correcto o justo.

Todas esas objeciones son correctas, pero se apartan de la cuestión fundamental. La cuestión fundamental radica en que las partes presentan argumentos que pretenden ser correctos, incluso aunque subjetivamente sólo estén persiguiendo sus propios intereses (Alexy, 1989a, 212). Pretenden, al menos, que sus argumentos serían aceptados por todos bajo condiciones ideales. Por ello contribuyen, como dice Habermas, «a un discurso que desde la perspectiva del juez sirve a la obtención de un juicio imparcial» (Habermas, 1998, 303). 
Este argumento ha sido recientemente atacado por Ulfrid Neumann. De acuerdo con Neumann, no basta concebir los argumentos de las partes como meras contribuciones que ayudan al juez a encontrar un juicio imparcial. Esto privaría a las partes de su estatus de participantes en los discursos y las reduciría a meras fuentes de información. Un procedimiento en el cual sólo un participante, el juez, decidiera c impusiera el argumento final y todos los demás sólo aportaran información no tendría una cstructura discursiva, sino monológica. Esto, de acuerdo con Neumann, contradice las ideas básicas de la teoría del discurso del Derecho (Neumann, 1996, 417 y s.).

Esta objeción subestima la complejidad necesaria para institucionalizar la racionalidad discursiva. Incluso aunque se esté de acuerdo con Neumann en que el acusado debe tener derecho a discutir todas las cuestiones jurídicamente relevantes de su caso con el juez (Neumann, 1996, 426), no se puede negar que es el tribunal quien, en última instancia, tiene que decidir y argumentar. Si el tribunal quiere decidir correctamente, tiene que oír todos los argumentos, lo que no es nada distinto del viejo audiatur et altera pars, y si la corrección de su decisión está sujeta a control, el tribunal deberá justificar su juicio ante los participantes y ante el público general y jurídico. Por ello, está sometido a los discursos ante los tribunales superiores, ante la profesión jurídica y ante el público. Todo ello, tomado conjuntamente, basta para interpretar los procedimientos judiciales en términos de teoría del discurso.

\section{Discurso moral, práctico general y jurídico}

\section{a) Discurso moral y argumentación jurídica}

El que la tesis del caso especial sea correcta o no depende de lo que se entienda por «discurso práctico general». Éste podría ser llamado el problema del genus proximum. Si se interpreta la expresión «discurso práctico general» como denotativa del discurso moral tal como lo define Habermas, la tesis del caso especial puede ser fácilmente considerada errónea. El discurso moral en el sentido de Habermas se refiere a la universalización y sólo a la universalización (Habermas, 1998, 221). La cuestión moral entra en juego si alguien pregunta qué normas

usólo pueden justificarse desde el punto de vista de si se tienen en cuenta por igual los intereses de todos [...] En el caso de las cuestiones morales la humanidad, o vale decir: la suposición de una república de ciudadanos del mundo, constituye el punto de referencia para la fundamentación de reglas que sean en interés de todos por igual» (Habermas, 1998, 173).

Resulta obvio que la argumentación jurídica está abierta no sólo a las razones morales definidas en este sentido, sino también a las razones ético-po- 
líticas y pragmáticas, tal y como son definidas por Habermas (Habermas, 1998, $222,302,356$ ). Las primeras se refieren al autoentendimiento colectivo implicado en las tradiciones y fuertes valoraciones (Habermas, 1998, 173); las segundas, a la adecuación de los medios para la consecución de ciertos objetivos y a la ponderación de los intereses y compromisos (Habermas, 1998, 173, 222 y 227).

Como cuestión no sólo de hecho, sino también como necesaria sistemáticamente, las razones éticas, al igual que las pragmáticas, desempeñan un rol indispensable en el razonamiento jurídico. Uno de los puntos de partida más importantes del razonamiento jurídico son las leyes que resultan del proceso democrático. En la toma de decisiones democrática esos tres tipos de razones son razones legítimas (Habermas, 1998, 173). Si la argumentación jurídica debe someterse a lo que ha sido decidido en el proceso democrático tiene que tomar en consideración los tres tipos de razones presupuestas por, o conectadas con, sus resultados.

\section{b) El concepto de discurso práctico general}

De este modo, la tesis de Habermas de que el discurso jurídico no debe ser concebido como un subconjunto de la argumentación moral, tal y como él la entiende (Habermas, 1998, 302), es obviamente verdadera. Pero esto no hace fracasar a la tesis del caso especial. De acuerdo con ella, el genus proximum del discurso jurídico no es el discurso moral en el sentido habermasiano, sino el discurso práctico general. Un discurso práctico general no es lo mismo que un discurso moral en el sentido de Habermas. Es un discurso en el cual las cuestiones y razones morales, éticas y pragmáticas están conectadas (Alexy, 1996b, 1033). Los discursos prácticos generales difieren de los discursos jurídicos en que no son dependientes de razones institucionales. Para la argumentación jurídica, las razones institucionales, como la ley y el precedente, son constitutivas, mientras que para la argumentación práctica general no lo son.

La formación de un concepto de discurso práctico que abarque a los argumentos morales, éticos y pragmáticos es, al mismo tiempo, algo sensato y necesario. Es sensato porque a menudo una argumentación puramente moral, esto es, una argumentación que sólo se ocupe de lo que es «igualmente bueno para todos los seres humanos» (Habermas, 1998, 221) no es suficiente para solucionar una cuestión práctica, es decir, una cuestión relativa a lo que debería ser hecho u omitido. En muchos casos los argumentos éticos y pragmáticos deben ser complementados con argumentos morales para conseguir la respuesta a una cuestión práctica. La dimensión pragmática comprende, de acuerdo con Habermas, la cuestión acerca de qué medios resultan adecuados para la consecución de ciertos objetivos, y esto conduce, en caso de que se produzca un conflicto entre objetivos, al problema de su ponderación (Habermas, 1998, 227). Cualquier problema práctico de cierta complejidad requiere tomar en 
consideración la relación entre medios y fines a objetivos y entre distintos objetivos. La adecuación es entonces un elemento neccsario del discurso práctico racional (Alexy, 1989a, 183 y ss.). De nuevo la justicia, entendida como lo que es igualmente bueno para todos, y la adecuación, incluso tomadas conjuntamente, no suelen resultar suficientes para decidir una cuestión práctica. Como ejemplos tendríamos los conflictos entre objetivos que no pueden ser resueltos únicamente por el criterio de lo igualmente bueno para todos. Se ha de tomar en cuenta entonces la dimensión ética para realizar una ponderación racional de los objetivos en conflicto sa la luz de las preferencias valorativas aceptadas" (Habermas, 1998, 227).

El discurso práctico general sería, dicho brevemente, un discurso que combinara los puntos de partida de la adecuación o utilidad, del valor o identidad y de la moralidad o justicia. Existiría tanto un orden prioritario como una relación de permeabilidad entre lo adecuado, lo bueno y lo justo.

\section{c) Las relaciones de prionidad entre los elementos del discurso práctico general}

La prioridad de lo bueno sobre lo adecuado resulta del hecho de que el mayor grado de adecuación de un medio para un fin no cuenta nada si el fin no tiene ningún valor en absoluto. La adecuación es atractiva sólo si el fin u objetivo tiene algún atractivo. La prioridad de lo justo sobre lo bueno es una cuestión mucho más difícil. Lo bueno como objeto del discurso ético expresa valores individuales, no universales y colectivos. Algo puede ser bueno o de valor para alguien sin ser bueno o de valor para todos. Lo justo representa el punto de vista moral universal. Su prioridad sólo puede ser justificada mostrando que cl punto de vista moral es necesario para todos. Esto puede hacerse reconstruyendo presuposiciones necesarias implícitas cn actos de habla elementales, como afirmar, preguntar y argumentar, que resultan inevitables o indispensables para todos. Aquí asumiremos que tal justificación de la validez universal del punto de vista moral es posible (cfr. Alexy, 1996c, 213 y ss.). Si esta suposición fuera verdadera, válida universalmente, implicaría la prioridad de lo justo sobre lo bueno.

\section{d) El discurso práctico general y la unidad del razonamiento práctico}

La prioridad es algo simple cuando lo que se ordena está claramente deslindado entre sí. Éste, sin embargo, no es el caso de lo justo y lo bueno. Se convierte en claro cuando el ámbito de lo justo no se reduce a los derechos humanos elementales, como el derecho a la vida, el derecho a no ser sometido a tortura y el derecho a no ser esclavizado. Estos derechos parecen pertenecer a lo que es igualmente bueno para todos, independientemente de las particulares concepciones de lo bueno. Pero si se concibe la justicia como un compromiso 
entre todas las cucstiones de distribución y retribución, entonces problemas como los relativos al Estado de bienestar y al castigo tienen que ser tratados como cuestiones de justicia. La respuesta a estas cuestiones dependerá de muchas razones. Entre ellas, los argumentos relativos a cómo comprenderse a uno mismo y a la comunidad en la que se vive desempeñan un papel esencial. Por ello, lo justo depende de lo bueno. Un cambio en la autocomprensión o la interpretación de la tradición en la que alguien ha sido educado (cfr. Alexy, 1989a, 199 y s.) pucdc implicar un cambio en su concepción de la justicia. Todo esto muestra que el discurso práctico general no es una simple mezcla o combinación, sino una conexión sistemáticamente necesaria que expresa la unidad sustancial de la razón práctica. Éste es el fundamento de la tesis del caso especial.

\section{Lo general y lo específico}

Incluso si se está de acuerdo en que la tesis del caso especial no se refiere a la moral, sino al discurso práctico general y en que, en principio, el discurso práctico general podría ser un genus proximum genuino, ya que es algo más que una mera mezcla o combinación de elementos pragmáticos, éticos y morales, se puede seguir insistiendo en que la tesis del caso especial está equivocada. Basta con decir que los argumentos prácticos generales cambian esencialmente su carácter o naturaleza cuando se emplean en contextos jurídicos. Dejan de ser argumentos generales y adquieren algo específicamente jurídico. Son, usando una expresión de Habermas (Habermas, 1998, 275), kimpregnados" por el Derecho*.

\section{a) «Otro modo de validez»y «cambio en el significado»}

Varias afirmaciones de Habermas apuntan en este sentido. En su opinión, la «emigración de los contenidos morales al interior del Derecho» no significa que estos contenidos continúen siendo simplemente contenidos morales. Ellos están «dotados de otro modo de validez» (Habermas, 1998, 276). Esto resulta bastante plausible por lo que se refiere a la dimensión de validez. Así, por ejemplo, un derecho moral, al conseguir validez jurídica además de validez moral, se transforma en un derecho fundamental como parte de una constitución. Pero Habermas se refiere no sólo a la dimensión de validez, y así afirma que:

* En el original inglés el autor llama la atención sobre el hecho de que esta expresión no ha sido traducida literalmente en la edición inglesa del libro de Habermas (cfr. Habermas, 1992, 252 , y 1996, 205). Sí lo ha sido, sin embargo, en la edición castellana que aquí citamos [N. de la T.]. 
«los contenidos morales, cuando son traducidos al código jurídico, experimentan un sistemático cambio en su significado, que les viene impuesto por la forma jurídicas (Habermas, 1998, 274).

Parece que Habermas quiere sostener que la transformación de contenidos morales en Derecho o su empleo en el mismo afecta no sólo a la dimensión de validez, sino también a la dimensión material. Esto se corresponde con la tesis de que los díscursos jurídicos están insertos en el sistema jurídico desde el principio y

«no constituyen casos especiales de argumentaciones morales que, en virtud de su vinculación al Derecho existente, se reduzcan a un subconjunto de lo moralmente obligatorio o permitido. Antes se refieren de raiz y desde el principio al Derecho democráticamente establecido y [...] no sólo se refieren desde el principio a normas jurídicas, sino que quedan ellos mismos insertas en el sistema jurídicon (Habermas, 1998, 306).

La cuestión es si los argumentos morales, al igual que otros argumentos del discurso práctico general, hacen peligrar tanto su carácter o naturaleza cuando se emplean en el discurso jurídico, que llegan a derribar la tesis del caso especial.

\section{b) El presupuesto del subconjunto}

Habermas adscribe a la tesis del caso especial dos presupuestos problemáticos, pero, afortunadamente, no neccsariamente conectados con ella. El primero puede ser llamado el presupuesto del subconjunto; el segundo, el presupuesto de la especificidad. De acuerdo con el primero, la tesis del caso especial diría que los discursos jurídicos son discursos morales «que, en virtud de su vinculación al Derecho vigente, se [reducen] a un subconjunto de lo moralmente obligatorio o permitido» (Habermas, 1998, 306). Esto se corresponde con la visión de que la argumentación juridica puede llegar hasta el punto en el que los argumentos específicamente jurídicos ya no estén disponibles. Es exactamente en este punto donde la argumentación práctica general debe intervenir. Ambas versiones del "presupuesto del subconjunto» son incompatibles con la idea de que, en la argumentación jurídica racional los argumentos específicamente jurídicos y los argumentos prácticos generales se combinan en todos los niveles y se aplican conjuntamente (Alcxy, 1989a, 271 y ss., 277 y s.). A esto se le podría llamar el presupuesto de la integración. La differentia specifica del discurso jurídico no viene dada únicamente por su restricción por el Derecho válidamente imperante, sino también por su integración en el sistema jurídico. 


\section{c) El presupuesto de la especificidad}

El segundo presupuesto problemático que Habermas adscribe a la tesis del caso especial es el presupuesto de la especificidad. De acuerdo con él, a la tesis del caso especial se le exige que muestre que las reglas y formas especiales de la argumentación jurídica «no hacen más que especificar las condiciones procedimentales gencrales de los discursos práctico-morales en lo tocante a vinculación al derecho vigente» (Habermas, 1998, 303). Nunca será posible satisfacer esta exigencia. Pero esto no perjudica a la tesis del caso especial. Existen algunas reglas y formas del discurso jurídico que de hecho se corresponden con las del discurso práctico general (Alexy, 1989a, 275 y ss.), pero el que no todas ellas lo hagan es no sólo inocuo, sino también necesario. El discurso jurídico está definido esencialmente por el empleo de razones autoritativas. Los argumentos lingüísticos, genéticos y sistemáticos nos ayudan a construir el rasgo de la vinculación de la argumentación jurídica a la autoridad, que resulta indispensable para la tesis del caso especial. Por esta razón, no es cierto que para hacer del discurso jurídico un caso especial del discurso práctico general todas las reglas y formas específicas del discurso jurídico tengan que ser casos especiales de las reglas y formas del discurso práctico general. Más bien sería correcto lo opuesto.

\section{d) Derecho injusto e irrazonable}

Se podría admitir todo esto y, aun así, insistir en que una stesis del caso especial» que evite el presupuesto del subconjunto y el de la especificidad no seria ya una tesis del caso especial.

El presupuesto de la especificidad se ha mostrado incompatible con el carácter autoritativo del razonamiento jurídico, que cxige reglas y formas de la argumentación jurídica que no sean casos especiales de reglas y formas del discurso práctico general. Es justamente este carácter autoritativo del razonamiento jurídico el que ha llevado a varios autores a pensar que el discurso jurídico no es un caso especial del discurso práctico general, sino algo cualitativamente distinto, un aliud (Neumann, 1986, 90; Braun, 1988, 259). Como una especie de piedra de toque podríamos citar una ley injusta o irrazonable, que sólo permitiera una decisión injusta o irrazonable (Neumann, 1986, 90). Habermas argumenta que en tales casos el presupuesto de la «consonancia entre el Derecho y la moralidad» que cree implícito en la tesis del caso especial «tiene también la desagradable consecuencia no sólo de relativizar la rectitud de la decisión jurídica, sino de ponerla como tal en cuestión» (Habermas, $1998,304)$. La razón para ello será que «las pretensiones de validez vienen codificadas binariamente y no admiten un más o un menos" (Habermas, 1998, 304). 
Para responder a esta objeción deben hacerse dos distinciones. La primera es la distinción entre dos aspectos que se encuentran combinados en la pretensión de corrección nccesariamente conectada con las decisiones judiciales (Alexy, 1989, 178 y ss.). El primer aspecto es la pretensión de que la decisión está correctamente justificada si se presupone el Derecho establecido, cualquiera que éste sea. El segundo aspecto es la pretensión de que el Derecho establecido, en el cual se basa la decisión, es justo y razonable. Ambos aspectos están contenidos cn la pretensión de corrección que aparece en las decisiones judiciales. Las decisiones judiciales no sólo pretenden ser correctas en el esquema del orden jurídico válidamente establecido, sino también pretenden ser correctas en cuanto decisión jurídica. Una decisión judicial que aplique correctamente una ley injusta o irrazonable no satisface en todos los aspectos la pretensión de corrección que ella despierta. Si la ley injusta o irrazonable es juridicamente válida, la decisión basada en ella también será jurídicamente válida, y en muchos, si no en la mayoría, de los casos, los principios de certeza jurídica, de división de poderes y de democracia exigirán al juez que cuando no haya margen para la interpretación, siga incluso las leyes injustas o irrazonables, de manera que su decisión será correcta bajo las circunstancias dadas, por desafortunadas que éstas sean. Pero, sin embargo, la decisión no es jurídicamente perfecta; está impregnada por la imperfección de la ley (Alexy, 1989a, 316-317).

La segunda distinción es la que surge entre una pretensión y su cumplimiento. La tesis del caso especial no asume que de hecho siempre exista una «consonancia entre moral y Derecho» (Habermas, 1998, 304). Sólo dice que tal consonancia está siempre implícita en las pretensiones del Derecho (Pavlokos, 1998, 148, 151 y s.). Tales pretensiones sólo tienen débiles consecuencias, aunque de largo alcance. Lo ponen todo bajo una luz diferente. Las decisiones judiciales injustas ya no podrán ser consideradas sólo moralmente controvertibles y, sin embargo, jurídicamente perfectas. Ellas serán también jurídicamente defectuosas. De este modo, el Derecho no sólo está abierto a la crítica moral desde fuera. La dimensión crítica se resitúa completamente dentro del propio Derecho.

La tesis de Habermas de que la corrección o rectitud de las decisiones jurídicas no es sólo relativizada, sino también cuestionada por una legislación injusta o irrazonable adopta significados muy diferentes, según se refiera a las pretensiones o a su cumplimiento. Si se refiere a las pretensiones, nada es relativizado; el mero no cumplimiento no pone en cuestión las pretensiones. Si se refiere al cumplimiento, su corrección o rectitud sí que es relativizada. Pero esto tampoco pone nada en cuestión, porque la tesis del caso especial sólo cxige las pretensiones y no su cumplimiento. 


\section{e) La integración de los argumentos y la institucionalización de la razón práctica}

Nos queda todavía la duda de si la sustitución del presupuesto del subconjunto por el presupuesto de la integración no priva a la tesis del caso especial de su base. Se podría argumentar que la integración de los argumentos prácticos generales en el contexto de los argumentos juridicos modifica su carácter o naturaleza. Si esto es cierto, la integración de los argumentos prácticos generales en un contexto jurídico implicará algo así como «otro modo de validez» (Habermas, 1998, 276), un «cambio en su significado, que les viene impuesto por la forma jurídica» (Habermas, 1998, 274), o una "dimensión de validez [...] mucho más complcja» (Habermas, 1998, 305). Si los argumentos prácticos generales cambian su carácter o naturaleza al ser integrados en contextos juridicos, el discurso práctico general ya no será el genus proximum del discurso jurídico y la tesis del caso especial se derrumbaría.

La integración de los argumentos prácticos generales en el contexto jurídico puede concebirse de dos maneras. La primera es coherentista; la segunda, procedimental. La visión coherentista más radical es la del holismo jurídico. De acuerdo con ella, todas las premisas ya forman parte de, o están ocultas en, el sistema jurídico y sólo necesitan ser descubiertas. Esta idea siempre ha resultado fascinante para los juristas por su promesa de total autonomía para el Derecho; ofrecería una solución perfecta al problema de legitimación del proceso de toma de decisiones judiciales. En una democracia, por ejemplo, haría posible remontar completamente cada decision jurídica hasta lo que ha sido promulgado en el proceso de legislación democrática, La máxima habermasiana de que los discursos jurídicos «se refieren de raiz y desde el principio al derecho democráticamente establecido» (Habermas, 1998, 306) sería sobradamente satisfecha. Sin embargo, la idea del holismo jurídico en forma de coherencia perfccta o ideal no es realizable, y Habermas es bastante claro al respecto: «la orientación por tan exigente ideal acabaría desbordando incluso a la administración de justicia más profesionalizada» (Habermas, 1998, 292). Cada fórmula sugerida por la precisión de esta idea resulta estar abicrta y depender de ser completada con valores y normas que todavía no están incluidos en lo ya establecido como Derecho válido. Si se adopta la idea hermenéutica de la estructura circular entre la precomprensión y el texto, entre la parte y el todo, y entre la norma y los hechos del caso, o la exigencia de que la aplicación racional de las normas tome en consideración todos los hechos del caso y todas las normas relevantes (Günther, 1993, 151), o la máxima de sentido común para buscar semejanzas, siempre se obtiene algo muy razonable pero también muy incompleto, y por tanto, necesitado de ser complementado de antemano (Dwars, 1992, 57 y s.; Alexy, 1993, 160 y ss.; Alexy, 1995, 75 y ss.). Del mismo modo que las normas no pueden ser aplicadas por sí mismas al 
sistema juridico, tampoco pueden producir coherencia. Para conseguirla se necesitan personas y procedimientos que suministren nuevos contenidos.

Esto nos lleva al segundo modo de integración de los argumentos prácticos generales en el contexto jurídico: cl procedimental. Es un problema grave y general de la teoría de la argumentación el si un argumento cambia su carácter o naturaleza al cambiar de contexto. Podemos asumir que la solución a este problema dependerá de lo que se entienda por cambio de carácter o naturaleza de un argumento. El fundamento de la comprensión de este concepto será de nuevo la idea de la unidad de la razón práctica. De acuerdo con esta idea, el sistema jurídico de un Estado democrático constitucional es un intento de institucionalizar la razón práctica. La razón práctica justifica la existencia del sistema jurídico como tal y de sus estructuras básicas; tiene que estar presente en los procedimientos de formación de la opinión - y voluntad- democrática para que sus resultados sean legítimos y debe ser empleada en la argumentación jurídica para satisfacer la pretensión de corrección que en ella surge. Los argumentos prácticos generales tienen que estar inmersos en todas las instituciones para que no se pierdan las raíces de esas instituciones en la razón práctica. Los argumentos prácticos generales son argumentos no institucionales. Los argumentos no institucionales que están inmersos en las instituciones pueden ser insertados, integrados y especificados tanto como se quiera, siempre y cuando continúen conservando lo que es esencial para ese tipo de argumentos: su carácter libre y no institucional. Ésta no es la única razón a favor de la tesis del caso especial, pero quizás sea la última.

\section{BIBLIOGRAFIA}

AIfXY, Robert (1989a): Teoría de la argumentación juridica, trad. de Manuel Atienza e Isabel Espejo, Centro de Estudios Constitucionales, Madrid.

- (1989b): «On Necessary Relations Between Law and Morality», Ratio Luris, 2: 167-183.

- (1993): «Justification and Application of Norms», Ratio Iuris, 6: 157-70.

- (1995): «Juristische Interpretation», en Robert Alexy, Recht, Vernunft, Diskurs, 71-92, Francfort, Suhrkamp.

- (1996a): Theorie der juristischen Argumentation, $3 .^{\mathrm{a}}$ ed., Francfort, Suhrkamp.

- (1996b): «Jürgen Habermas's Theory of Legal Discourse», Cardozo Law Review, 17: 1027.1034 .

- (1996c): «Discourse Theory and Human Rights», Ratio luris, 9: 209-235.

BRAUN, Carl (1988): «Diskurstheoretische Normenbegründung in der Rechtswissenschaft $\gg$, Rechstheorie, 19: 238-261.

Dwars, Ingrid (1992): «Application Discourse and Special Case-Thesis», Ratio Iuris, 5: 67-78.

GüNTHER, Klaus (1993): «Critical Remarks on Robert Alexy's "Special-Case Thesis"», Ratio luris, 6: 143-156.

HABERMAS, Jürgen (1992): Faktizität und Geltung, Francfort, Suhrkamp.

- (1996): Between Facts and Norms, trad. W. Rehg, Cambridge, Polity Press. 
- (1998): Facticidad y validez, trad. Manuel Jiménez Redondo, Trotta, Madrid.

Hart, H. L. A. (1994): The Concept of Law, 2.* ed., Oxford, Clarendon Press (trad. castellana de Jenaro Carrió: El concepto de derecho, Abeledo-Perrot, Buenos Aires).

KAUFMANN, Arthur (1989): aLaBt sich die Hauptverhandlung in Strafsachen als rationales Diskurs auffassen?), en Dogmatik und Praxis des Sirafverfahrens, ed. H. Jung y H. Müller-Dietz, 15-24. Colonia, Berlín, Bonn y Munich, Heymanns.

NeUMAnN, Ulfrid (1986): Juristische Argumentationslehre, Darmstadt, Wissenschaftliche Buchgesellschaft.

- (1996): «Zur Interpretation des forensischen Diskurses in der Rechtsphilosophie von Jürgen Habermas", Rechtstheorie, 27: 415-426.

Pavlakos, Georgios (1998): «The Special Case Thesis. An Assessment of R. Alexy's Discursive Theory of Law», Ratio luris, 11: 126-154. 